\title{
Aportaciones a la flora briofítica de los bosques tropicales y subtropicales de la Argentina (Bryophyta, Musci)
}

\author{
Esther Fuertes ${ }^{1}$, Carmen Prada ${ }^{1}$ y Cristina Rolleri ${ }^{2}$
}

Resumen: Fuertes, E.; Prada, C. \& Rolleri, C. 2015. Aportaciones a la flora briofítica de los bosques y selvas tropicales y subtropicales de la Argentina (Bryophyta, Musci). Bot. Complut. 39: 49-61.

Se presenta una lista de 32 especies de musgos pertenecientes a 15 familias: Brachytheciaceae, Cryphaeaceae, Entodontaceae, Hedwigiaceae, Hypnaceae, Leptodontaceae, Meteoriaceae, Neckeraceae, Pilotrichaceae, Polytrichaceae, Pterobryaceae, Racopilaceae, Rigodiaceae, Stereophyllaceae y Trachypodaceae, recolectados en los bosques montanos de las Yungas del NO de la Argentina (Jujuy, Salta, Catamarca y Tucumán) y en las selvas del NE del país (Misiones). Ocho especies: Atrichum polycarpum, Chryso-hypnum elegantulum, Pilosium chlorophyllum, Pilotrichella flexilis, Porotrichodendron lindigii, Pseudotrachypus martinicensis, Steerecleus scariosus y Thamnobryum fasciculatum son nuevas aportaciones para la flora briológica argentina. Braunia imberbis y Squamidium brasiliense son nuevas citas para la brioflora de la provincia de Catamarca; Porotrichodendron superbum es novedad para Salta mientras que Forsstroemia coronata es primera cita para las provincias de Catamarca y Jujuy. Aerolindigia capillacea, Braunia reflexifolia, Chryso-hypnum diminutivum, Meteorium deppei y Meteoridium remotifolium son cinco nuevas citas para la brioflora de la provincia de Jujuy y Schoenobryum concavifolium es nueva para la flora briofítica de Misiones.

La mayoría de las especies estudiadas son más frecuentes en las Yungas (NO argentino); otras tienen una distribución disyunta en las Yungas y en la selva Paranaense, y otras están restringidas a la selva Paranaense del NE de la Argentina. Se actualiza la taxonomía de especies y se incluyen comentarios sobre antecedentes bibliográficos, ecología y corología de cada taxón.

Palabras clave: Bryophyta, ecología, corología, bosques subtropicales y tropicales, norte de Argentina.

Abstract: Fuertes, E.; Prada, C. \& Rolleri, C. H. 2015. Contributions to the bryological flora of the tropical and subtropical forests from the Argentina (Bryophyta, Musci). Bot. Complut. 39: 49-61.

The authors present here a list of 32 mosses belonging to 15 families: Brachytheciaceae, Cryphaeaceae, Entodontaceae, Hedwigiaceae, Hypnaceae, Leptodontaceae, Meteoriaceae, Neckeraceae, Pilotrichaceae, Polytrichaceae, Pterobryaceae, Racopilaceae, Rigodiaceae, Stereophyllaceae, and Trachypodaceae, all collected in the mountainous forests of the Yungas of the NW of the Argentina (Jujuy, Salta, Catamarca and Tucumán provinces), and also in the rainforests from the NE of the country (Misiones province). Eight species: Atrichum polycarpum, Chrysohypnum elegantulum, Pilosium chlorophyllum, Pilotrichella flexilis, Porotrichodendron lindigii, Pseudotrachypus martinicensis, Steerecleus scariosus, and Thamnobryum fasciculatum are new records for the bryologic flora from Argentina. Braunia imberbis and Squamidium brasiliense are two new records for the bryophytic flora of the Catamarca province; Porotrichodendron superbum is new for the Salta province, while Forsstroemia coronata is the first record for the Catamarca and Jujuy provinces. Aerolindigia capillacea, Braunia reflexifolia, Chryso-hypnum diminutivum, Meteorium deppei and Meteoridium remotifolium are five new citations for the Jujuy province, and Schoenobryum concavifolium is new for the bryophytic flora of the Misiones province.

Many studied species occur more frequently in the Yungas than in the NE rainforests; others show separated distribution but live in both areas, the Yungas and Paranaense area, and others are more or less restricted to the Paranaense rainforest of the NE of Argentina. The taxonomy of species is updated, and comments are included on bibliographical precedents, ecology and chorology of each taxon.

Key words: Bryophyta, ecology, chorology, subtropical and tropical forests, north of Argentina.

\section{INTRODUCCION}

Se presenta un catálogo de musgos recolectados en el curso de sucesivos viajes de campo realizados al NO y NE de la Argentina. Constituye la continuación de otras apor- taciones realizadas por Matteri (2003) y, desde 2007 en adelante, a la flora briofítica de la Argentina por Fuertes \& Hedenäs (2007), Fuertes (2008), Fuertes \& Oliván (2008), Fuertes \& Rodríguez (2008), Koponen \& Fuertes (2010) y Fuertes et al. (2012) entre otras.

\footnotetext{
${ }^{1}$ Departamento de Biología Vegetal I, Facultad de Biología Universidad Complutense de Madrid, E-28040 Madrid (España). efuertes@ucm.es

${ }^{2}$ Laboratorio de Estudios de Anatomía Vegetal Evolutiva y Sistemática, 64-3, 1904DZB, La Plata, Facultad de Ciencias Naturales y Museo, Universidad Nacional de La Plata, Argentina.

Recibido: 28 octubre 2014. Aceptado: 7 enero 2015.
} 
El área de estudio está incluida en los dominios biogeográficos Amazónico y Chaqueño (Cabrera 1976), cuya vegetación dominante está formada por bosques y selvas húmedos e hiperhúmedos de la parte septentrional de la Argentina, representada por las Yungas, al NO, principalmente en las provincias de Jujuy, Salta, Catamarca y Tucumán ( Parque Nacional de Calilegua, las Lagunas de Yala, Parque provincial El Potrero de Yala en Jujuy; Reserva de la Quebrada de Los Sosa y la Sierra de Ascochinga en Tucumán; Reserva Nacional El Nogalar, Parque Nacional de Baritú, Reserva de San Andrés en Salta y las áreas protegidas de la selva Paranaense, al NE, una extensión de la selva Amazónica que ocupa entre otros la Reserva de la Biosfera Yabotí, el Parque Nacional de Mocconá, el Parque Nacional Iguazú, el Parque Nacional de Urugua-í y el Parque Regional Cruce Caballero en la provincia de Misiones.

El objetivo de este trabajo es dar a conocer la presencia de musgos que son nuevas aportaciones a la flora de Argentina 0 sus provincias y actualizar el conocimiento de la flora briológica desde el punto de vista corológico.

\section{MATERIALES Y MÉTODOS}

En el catálogo, las familias se ordenan alfabéticamente. Para cada taxón se suministran los sinónimos más comunes, las referencias bibliográficas previas y se describe la ecología y corología general. El símbolo $\bullet$ indica una nueva aportación a la flora briológica de Argentina; el símbolo * indica primera cita provincial y el símbolo ** segunda cita provincial.

Para la sistemática se ha seguido el criterio de Goffinet et al. (2008); mientras que para la identificación de los taxones se han utilizado las claves de Churchill \& Linares (1995), Gradstein et al. (2001), Sharp et al. (1993) y las monografías de géneros citadas en el texto. Todos los pliegos están registrados en el Herbario de la Facultad de Ciencias Biológicas de la Universidad Complutense de Madrid (MACB); los duplicados disponibles se han donado a diferentes herbarios citados en el texto: Museo de Historia Natural de Buenos Aires (BA), Real Jardín Botánico de Madrid (MA), Missouri Botanical Garden (MO), New York Botanical Garden (NY), Swedish Natural Museum (S), entre otros. La distribución geográfica de los taxones en la Argentina se resume en la Tabla 1.

\section{RESULTADOS}

\section{Brachytheciaceae}

\section{Aerolindigia capillacea (Hornsch.) Menzel}

Pilotrichum capillaceum Hornsch. 1840.- Lindigia capillacea (Hornsch.) Hampe 1868.- Lindigia lorentzii Müll. Hal. 1879.Rhynchostegiella capillacea (Hornsch.) Visnadi \& Allen 1991.
*Jujuy: Ledesma, P. Nac. de Calilegua, Paraje Tres Cruces, 234' S $64^{\circ} 55^{\prime} \mathrm{W}, 1060 \mathrm{~m}, 29-06-2007$, Fuertes \& Prada (MACB 104466). Tucumán: Tafí del Valle, Reserva de la Quebrada de Los Sosa, 26045'S 654' W, 700 m, 29-04-2007, Prada \& Gallardo, BA, MACB 104465.

Citas previas. - La primera referencia de Aerolindigia capillacea en Argentina corresponde a Müller (18781879), quien determina los especímenes recolectados por Lorentz en 1873 en Salta (San Andrés) y en 1874 en Tucumán (Siambón) como Lindigia lorentzii; posteriormente fue recolectada en Tucumán (Quebrada de Lules) por Garolera en 1946 (Menzel 1991).

Ecología y distribución.- Epífita, colgante en troncos y ramas; se comporta como especie mesófila, higrófila y esciófila. Pantropical (Menzel 1991), con disyunción afro-americana: África (Madagascar, Ruanda, Uganda, Zaire), Norteamérica (México), Centroamérica (Costa Rica, Guatemala, Panamá), Sudamérica (Colombia, Venezuela, Ecuador, Perú, Brasil). En Argentina su área de distribución está restringida a la parte NO, en los bosques montanos de las Yungas. En esta aportación se cita por primera vez para la provincia de Jujuy (Tabla 1).

\section{Squamidium brasiliense (Hornsch.) Broth.}

Antitrichia brasiliensis Hornsch. 1840.- Neckera brasiliensis (Hornsch.) C. Müll. 1850._-Meteorium brasiliense (Hornsch.) Mitt. 1859.- Pilotrichum brasiliense (Hornsch.) Hampe 1865.-Pterobryum brasiliense (Hornsch.) Mitt. 1869.

*Catamarca: El Alto, Sierra de Ancasti, camino de El Portezuelo a El Alto, $28^{\circ} 27^{\prime} \mathrm{S} 63^{\circ} 35^{\prime} \mathrm{W}, 1340 \mathrm{~m}, 16-09-2010$, Fuertes, BA, MA, MACB 101676, 104454 y 102247, MO, NY. Jujuy: Ledesma, P. Nac. de Calilegua, Paraje El Tigre, 234ㄴ'S 645ㄴ, 1550-1700 m, 16-6-2008, Fuertes \& Prada, MACB 101041. Manuel Belgrano, subida a las Lagunas de Yala, en bosques de nogal criollo (Juglans australis) y aliso (Alnus acuminata), 240ㄱ'S 652' W, 1250-2000 m, 14-06-2008, Fuertes \& Prada, MACB 101042. Salta: Capital, Barranca de San Lorenzo, carretera a Vaqueros, bosques de cebil colorado (Anadenanthera colubrina), $24^{\circ} 44^{\prime} \mathrm{S} 65^{\circ} 29^{\prime} \mathrm{W}, 1390 \mathrm{~m}, 30-07-2007$, Prada \& Gallardo, BA, MACB 101043, MO; El Alto, paraje Ojo del Agua, camino de Salta a Jujuy por

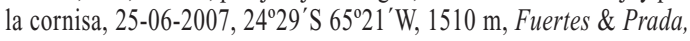
MACB 101040, MO; ibidem, paraje el Descargadero, bosque montano de alisos (Alnus acuminata), entre Salta y Jujuy, por la cornisa, $1475 \mathrm{~m}$, 25-06-2007, Fuertes \& Prada, MACB 101038, M0; Orán, Reserva de San Andrés, paraje de Las Maromas, selva pedemontana con helechos arborescentes, $23^{\circ} 07^{\prime} \mathrm{S} 64^{\circ} 37^{\prime} \mathrm{W}, 590-950 \mathrm{~m}, 17-06-2008$, Fuertes, MACB 101039. Tucumán: Tafí del Valle, El Nogalar, $26^{\circ} 45^{\prime} \mathrm{S} 65^{\circ} 21^{\prime} \mathrm{W}$, $1500 \mathrm{~m}, 20-06-2007$, Fuertes \& Prada, MACB 101036; ibidem alrededores de Villa Nougés, 1450 m, 20-06-2007, Fuertes \& Prada, MACB

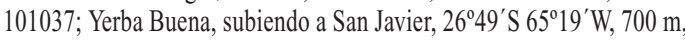
30-04-2007, Prada \& Gallardo, MACB 101035, MO. Quebrada de Los Sosa, paraje La Heladera, 1350 m, 29-04-2007, Prada \&. Gallardo, MO.

Citas previas.-Müller (1878-1879) describe los especimenes recolectados por Lorentz en Salta (San Andrés) 
Tabla 1

Distribución de las especies en las provincias biogeográficas de Argentina (Cabrera 1976). BA: Buenos Aires, CA: Catamarca, CH: Chaco, C0: Córdoba, CRR: Corrientes, F0: Formosa, JU: Jujuy, MI: Misiones, SA: Salta, TU: Tucumán. Novedad nacional $(\bullet)$, novedad provincial $(*)$, segunda cita provincial $(* *)$

\begin{tabular}{|c|c|c|c|c|}
\hline Especies & $\begin{array}{c}\text { Prov. } \\
\text { Yungueña }\end{array}$ & $\begin{array}{c}\text { Prov. } \\
\text { Chaqueña }\end{array}$ & $\begin{array}{c}\text { Prov. } \\
\text { Pampeana }\end{array}$ & $\begin{array}{c}\text { Prov. } \\
\text { Paranaense }\end{array}$ \\
\hline Aerolindigia capillacea & *JU, SA, TU & & & \\
\hline - Atrichum polycarpum & JU, SA, TU & & & \\
\hline Braunia exserta & **JU, TU & & & \\
\hline Braunia imberbis & ${ }^{*} \mathrm{CA}, \mathrm{SA}, \mathrm{TU}$ & & & \\
\hline Braunia reflexifolia & *JU, TU & & & \\
\hline Braunia tucumanensis & TU & & & \\
\hline Cryphaea racomitrioides & **JU, TU & & & \\
\hline Chryso-hypnum diminutivum & $* J U, S A$ & $\mathrm{CH}$ & & CRR, MI \\
\hline - Chryso-hypnum elegantulum & SA & & & \\
\hline Erythrodontium squarrosum & SA & & & CRR, MI \\
\hline Forsstroemia coronata & ${ }^{*} \mathrm{CA},{ }^{* J U}, \mathrm{SA}, \mathrm{TU}$ & $\mathrm{CH}, \mathrm{FO}$ & BA & CRR, MI, ER \\
\hline Juratzkaea argentinica & $* * \mathrm{SA}$ & $\mathrm{CO}$ & & \\
\hline Meteorium deppei & $* J U, * * S A, T U$ & & & CRR, MI \\
\hline Meteorium nugrescens & SA & & & CRR, MI \\
\hline Meteoridium remotifolium & *JU, SA, TU & & & $\mathrm{CRR}, * \mathrm{MI}$ \\
\hline Mittenothamnium reptans & SA, TU & & & CRR, MI \\
\hline - Pilosium chlorophyllum & JU, SA, TU & & & \\
\hline - Pilotrichella flexilis & & & & MI \\
\hline - Porotrichodendron lindigii & SA & & & \\
\hline P. superbum & $* \mathrm{SA},{ }^{* * \mathrm{TU}}$ & & & \\
\hline Porotrichum thieleanum & $* * \mathrm{SA}, * \mathrm{TU}$ & & & \\
\hline - Pseudotrachypus martinicensis & & & & MI \\
\hline Pterobyon densum & SA & & & \\
\hline Pterobryopsis stolonaceae & $\mathrm{JU},{ }^{*} \mathrm{SA}, \mathrm{TU}$ & & & CRR \\
\hline Racopilum tomentosum & **U, SA, TU & & & CRR, MI \\
\hline Rigodium toxarion & $* * \mathrm{TU}$ & & & \\
\hline Schoenobryum concavifolium & SA & & & *MI \\
\hline - Steerecleus scariosus & JU, SA, TU & & & \\
\hline Squamidium brasiliense & *CA, JU, SA, TU & & & MI \\
\hline Stereophyllum radiculosum & $* * \mathrm{JU}$ & FO & & \\
\hline Thamniopsis incurva & & & & MI \\
\hline - Thamnobryum fasciculatum & JU, SA, TU & & & MI \\
\hline
\end{tabular}

y en áreas serranas de Tucumán, en 1873, como Meteorium lorentzii. Hosseus (1940), Allen \& Crosby (1986) y Drehwald (1995) la citan en Misiones.

Ecología y distribución.-Epífita sobre troncos o colgante en las ramas de diferentes forófitos de los bosques tropicales. Paleo-neotropical: África y Sudamérica (Allen $\&$ Crosby 1986). En Argentina se distribuye en los bosques hiperhúmedos de clima cálido del Dominio Amazónico, en dos áreas disyuntas, las Yungas al NO y la pro- vincia Paranaense al NE. Es primera cita para los departamentos Ledesma y Manuel Belgrano (Jujuy) y para los departamentos Capital y El Alto (Salta).

- Steerecleus scariosus (Taylor) H. Robins

Hypnum scariosum Taylor 1846.-Rhynchostegium scariosum (Taylor) Jäger 1878.- Eurhynchiella scariosa (Taylor) Williams 1928.Eurhynchium scariosum (Taylor) Bartram 1946. 
Jujuy: Manuel Belgrano, subida a las Lagunas de Yala, suelos pedregosos en bosques de Juglans australis) y Alnus acuminata, $24^{\circ} 07^{\prime} \mathrm{S}$ 652'․ W, 1250-2000 m, 14-06-2008, Fuertes \& Prada, MACB 106363. Salta: El Alto, km 1642, paraje El Descargadero, por la ruta de la cornisa, $24^{\circ} 29^{\prime} \mathrm{S} 65^{\circ} 21^{\prime} \mathrm{W}, 1475 \mathrm{~m}, 25-06-2007$, Fuertes \& Prada, MACB 105504. El Alto, Paraje Ojo del Agua, ruta de la cornisa, 1510 m, 2506-2007, Fuertes \& Prada, BA, MACB 105503. Los Monteros, bosque subtropical serrano de robles, laureles, cebil colorado y lapachos perennifolios, entre la Quebrada de Scoipe y Los Laureles, $25^{\circ} 09^{\prime} \mathrm{S}$ $65^{\circ} 45^{\prime} \mathrm{W}, 1370 \mathrm{~m}, 15-6-2008$, Fuertes, Gallardo \& Prada, MACB 106728. Tucumán: Chicligasta, Sierra de Aconquija, bosque montano -las Yungas- con Alnus acuminata, suelo del bosque, $27^{\circ} 18^{\prime} \mathrm{S} 65^{\circ} 54^{\prime} \mathrm{W}$, 885 m, 22-6-2007, Fuertes \& Prada, MACB 106363.

Ecología y distribución.-Crece sobre raíces y base de troncos de forófitos lauroides, en bosques húmedos; se comporta como especie higrófila y esciófila. Neotropical. Se distribuye en Norteamérica (México), Centroamérica y Sudamérica (Colombia, Ecuador, Perú, Bolivia) (Robinson 1987) y Uruguay (Fuertes \& Marchesi 2013). Es nueva aportación a la brioflora argentina y su área está restringida a los bosques montanos de las Yungas, en el NO del país.

\section{Cryphaeaceae}

\section{Cryphaea rhacomitrioides Müll. Hal.}

Cryphaea rhacomitrioides var. fragilis Müll. Hal. 1881.

**Jujuy: Manuel Belgrano, subida a las lagunas de Yala, epífita en el bosque montano de Juglans australis y Alnus acuminata $24^{\circ} 07^{\prime} \mathrm{S}$ $65^{\circ} 22^{\prime} \mathrm{W}$, desde 1250-2000 m, 19-11-2005, Fuertes, MACB 104716. Tucumán: Yerba Buena, El Rulo, selva montana de Myrtaceae, 26049'S $65^{\circ} 19^{\prime} \mathrm{W}, 700$ m, 30-04-2007, Prada \& Gallardo, MA, MACB104715.

Citas previas.- Jujuy (Rao 2001), Tucumán (Rao 2001, Suárez \& Schiavone 2010) y recolectada en Tucumán por Churchill \& Schiavone en 1999 (Tropicos.org 2014).

Ecología y distribución.- Epífita en bosques húmedos de las Yungas, mesófila y orófila. Neotropical: Brasil y Argentina (Tropicos.org 2014).

\section{Schoenobryum concavifolium (Griff.) Gangulee}

Orthotrichum concavifolium Griff. 1842.- Cryphaea concavifolia (Griff.) Mitt. 1859.-Acrocryphaea concavifolia (Griff.) Bosch \& Sande Loc. 1864._-Pilotrichum concavifolium (Griff.) Müll. Hal. 1869._Acrocryphaea gardneri (Mitt.) Manuel 1999.

*Misiones: Guaraní, Reserva de la Biosfera Yabotí, selva tropical, con helechos arborescentes, a lo largo del arroyo Yabotí Guazú, pr. El So- berbio, $27^{\circ} 10^{\prime} \mathrm{S} 54^{\circ} 04^{\prime} \mathrm{W}, 410 \mathrm{~m}, 27-09-2007$, Fuertes \& Prada, BA, MACB 106719, NY.

Citas previas.- Salta (Herzog 1952, Suarez \& Schiavone 2010, Hübschmann 1986).

Ecología y distribución.-Epífita en troncos y ramas de diferentes forófitos, en bosques húmedos, densos y sombríos, mezclada con hepáticas foliosas (Jungermanniopsida). Se comporta como mesófila, higrófila y esciófila. Pantropical, en dos áreas disyuntas: Asia y América. En Sudamérica se extiende por Venezuela, Colombia, Ecuador, Perú, Bolivia, Argentina, Brasil (Tropicos.org 2014). Rara en Argentina, sólo se conoce en Salta; esta aportación es primera cita para Misiones.

\section{Entodontaceae}

\section{Erythrodontium squarrosum (Hampe) Paris}

Leptohymenium squarrosum Hampe 1862.-Entodon squarrosus (Hampe) Mitt. 1869.- Pterygynandrum squarrosum (Hampe) Hampe 1870 .

Misiones: Iguazú, P. Nac.Iguazú, selva atlántica en la ribera del Arroyo de Santo Domingo, junto a la ruta 101, 90 m, 12-09-2008, Fuertes, Oliván, Herrero, \& Prada, MACB104451; ibidem, entre la Estación

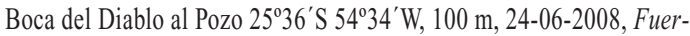
tes \& Prada, MACB 106721.

Citas previas.- Misiones (Herzog 1952, Hosseus 1940, Drehwald 1995), Corrientes (Hübschmann 1986) y Salta (Herzog 1952).

Ecología y distribución.-Epífita en troncos y ramas de bosques tropicales; mesófila, higrófila, esciófila. Tropical, con disyunción afro-americana. En América desde Norteamérica (México), Centroamérica hasta Sudamérica. En Argentina su área de distribución está restringida al Dominio Amazónico.

\section{Hedwigiaceae}

Braunia exserta Müll. Hal.

\footnotetext{
**Jujuy: Manuel Belgrano, subida a las lagunas de Yala, bosque montano de Juglans australis y Alnus acuminata, $24^{\circ} 07^{\prime} \mathrm{S} 65^{\circ} 22^{\prime} \mathrm{W}$, desde 1250-2000 m, 19-11-2005, Fuertes, BA, MA, MACB 104718, MO, NY.

Citas previas.- Jujuy y Tucumán (Biasuso 1992).

Ecología y distribución.-Epífita, rara vez saxícola, mesófila e higrófila. Neotropical, Sudamérica: Brasil, Argentina (Biasuso 1992).
} 


\section{Braunia imberbis (Sm.) N. J. Dalton \& D. G. Long}

Gymnostomun imberbe Sm. 1811.-Anictangium imberbe (Sm.) Hook. \& Tayl. 1818.- Hedwigidium imberbe (Sm.) Bruch \& Schimp. 1846.-Hedwigia imberbis (Sm.) Müll. Hal. 1850.

* Catamarca: Padín, carretera de La Viña a la Merced, en rocas y suelos del bosque, 2806'S 653' W, 1130 m, 15-09-2010, Fuertes, BA, MACB 106720, MO

Citas previas.-Tucumán (Müller 1878-1879); Salta (Tropicos.org, 2014).

Ecología y distribución.-Especie saxícola, meso-xerófila y fotófila. Pantropical, con áreas disyuntas en América, Europa y Australia (Tropicos.org 2014). En Argentina es nueva para la provincia de Catamarca; su área de distribución está restringida a la provincia Yungueña.

\section{Braunia reflexifolia (Müll. Hal.) E. B. Bartram}

Braunia exserta var. reflexifolia Müll. Hal. 1879.

*Jujuy: Manuel Belgrano, Parque provincial El Potrero de Yala, Laguna del Comedero, bosques montanos de nogal criollo y alisos, $24^{\circ} 10^{\prime} \mathrm{S}$ 652', 1950 m, 14-06-2005, Fuertes, MACB 104714. Tucumán: Chicligasta, Sierra de Aconquija, bosque montano -las Yungas- con Alnus acuminata, Paraje La Banderita, $27^{\circ} 18^{\prime} \mathrm{S} 65^{\circ} 54^{\prime} \mathrm{W}$, entre $1845-1860 \mathrm{~m}$, 22-06-2007, Fuertes \& Prada, BA, MACB 104719, 106729.

Citas previas.-Tucumán (Biasuso 1992).

Ecología y distribución.-Epifita, mesófila, orófila. Neotropical, Sudamérica: Brasil y Argentina (Biasuso 1992). Es nueva aportación para la provincia de Jujuy.

\section{Braunia tucumanensis Biasuso}

Tucumán: Yerba Buena, selva montana de Myrtaceae -yungas- subiendo a San Javier, 1350 m, 30-04-2007, Prada \& Gallardo, BA, MA, MACB 104717.

Citas previas.-Tucumán (Biasuso 1992).

Ecología y distribución.-Epífita en árboles y arbustos; se comporta como especie mesófila e higrófila. Neotropical, endemismo argentino. Esta aportación es novedad para el departamento Yerba Buena.

\section{Hypnaceae}

Chryso-hypnum diminutivum (Hampe) W. R. Buck

Hypnum diminutivum Hampe 1847.- Mittenothamnium diminutivum (Hampe) Jäger. 1878. - Microthamnium pringlei (Cardot) Broth. 1925.
*Jujuy: Ledesma, P. Nac.de Calilegua, selva montana de Myrtaceae camino de la Junta a la lagunilla, 23⒋ $44^{\prime} \mathrm{S} 64^{\circ} 55^{\prime} \mathrm{W}, 760 \mathrm{~m}, 29-06-2007$, Fuertes \& Prada, MACB 106324, 106707. Salta: Orán, paraje Los Naranjos, selva pedemontana $23^{\circ} 10^{\prime} \mathrm{S}^{\prime} 63^{\circ} 29^{\prime} \mathrm{W}, 400-650 \mathrm{~m}, 17-06-2008$, Fuertes, MACB 105489, NY; ibidem, Paraje de Las Maromas (con abundancia de Ficus maroma, epífita en helechos arborescentes, $23^{\circ} 07^{\prime} \mathrm{S} 64^{\circ} 37^{\prime} \mathrm{W}, 890-950 \mathrm{~m}, 16-06-2008$, Fuertes, Martínez \& Prada, MACB 105490.

Citas previas.-En las provincias de Chaco, Corrientes y Misiones (Drehwald 1995); en Salta, recolectada por Churchill \& Schiavone en 1999 (Tropicos.org 2014).

Ecología y distribución.- Epífita en troncos, tocones, a veces sobre rocas del sotobosque mezclada con otras muscíneas. Neotropical, en Sudamérica su área se extiende por Venezuela, Colombia, Ecuador y Perú, Argentina (Tropicos.org 2014) y Uruguay (Herter 1943). Esta aportación es novedad provincial (Jujuy) y en Salta amplía su área de distribución desde San Andrés hasta Los Naranjos y Río Blanquito de Santa Cruz.

\section{- Chryso-hypnum elegantulum (Hook.) Hampe}

Hypnum elegantulum Hook. 1818.-Microthamnium elegantulum (Hook.) Mitt. 1869.- Stereohypnum elegantulum (Hook.) M. Fleisch. 1908.- Mittenothamnium elegantulum (Hook.) Cardot 1910.-Rhizohypnum elegantulum (Hook.) Herzog 1916.

Salta: Orán, Reserva de San Andrés, paraje Los Naranjos, selva pedemontana con abundancia de Ficus maroma, $23^{\circ} 10^{\prime} \mathrm{S}^{\prime} 63^{\circ} 29^{\prime} \mathrm{W}, 400$ 650 m, 16-06-2008, Fuertes, MACB 105488; ibidem, paraje de Las Maromas, selva pedemontana con helechos arborescentes, $23^{\circ} 07^{\prime} \mathrm{S}$ $64^{\circ} 37^{\prime} \mathrm{W}$, entre 890-950 m, 16-06-2008, Fuertes, Martínez \& Prada, MACB 105490.

Ecología y distribución.-Epífita hacia la base de forófitos lauroides, en las Yungas, se comporta como especie mesófila, higrófila y esciófila. Neotropical, se extiende de México a Sudamérica (Nishimura \& Hisatsugu 1994), Bolivia (Tropicos.org 2014), Venezuela (Pursell 1973), y Argentina (esta aportación).

\section{Mittenothamnium reptans (Hedw.) Cardot}

Hypnum reptans Hedw. 1801.-Chryso-hypnum pendulinum Hampe 1870.-Eurhynchium mexicanum Besch 1872.-Microthamnium mexicanum (Besch.) Jäg. 1878.- Mittenothamnium mexicanum (Besch.) Cardot 1911

Salta: El Alto, laguna junto al Dique de Campo Alegre, pr. Alto de la

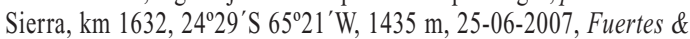
Prada, BA, MACB 106727, MO; Santa Victoria, Reserva Nacional El 
Nogalar, pr. Los Toldos, bosque montano con Alnus acuminata, $22^{\circ} 18^{\prime} \mathrm{S}$

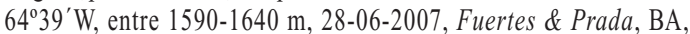
MACB106326. Tucumán: Chicligasta, Sierra de Aconquija, selva montana de Myrtaceae con alisos, subiendo a La Banderita, $27^{\circ} 20^{\prime} \mathrm{S}$ $65^{\circ} 48^{\prime} \mathrm{W}$, entre 1845-1860 m, 22-06-2007, Fuertes \& Prada, BA, MACB 105498, MO, NY.

Citas previas.-Corrientes, Misiones (Drehwald 1995); recolectada en Salta por Price, Biasuso, Churchill, Magombo \& Schiavone en 1999 y en Tucumán por Churchill \& Schiavone en 1999 (Tropicos.org 2014).

Ecología y distribución.-Epífita en troncos y base de troncos, tocones, a veces suelos y rocas húmedas del sotobosque; mesófila, higrófila, esciófila. Paleotropical y neotropical. En África (Etiopía, Kenia, Camerún, Tanzania, Zimbabue, Sudáfrica); en América: México, Centroamérica y Sudamérica: Venezuela, Colombia, Ecuador, Perú, Bolivia, Argentina (Tropicos.org 2014). Esta aportación incluye materiales de localidades de los departamentos de Santa Victoria (Salta) y Chicligasta (Tucumán).

\section{Leptodontaceae}

\section{Forsstroemia coronata (Mont.) Paris}

Leptodon coronatus Mont 1838.- Neckera coronata (Mont.) Müll. Hal. 1850.-Leucodon coronatus (Mont.) Mitt. 1869.-Lasia coronata (Mont.) Müll. Hal. 1879._- Dusenia coronata (Mont.) Müll. Hal. 1917.

*Catamarca: Padín, carretera de La Viña a la Merced, 2806'S $65^{\circ} 37^{\prime} \mathrm{W}, 1139 \mathrm{~m}, 15-09-2010$, Fuertes \& Prada, BA, MACB 106708,

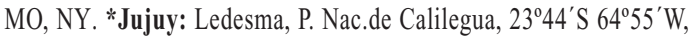
selva montana de Myrtaceae, camino de la Junta a la lagunilla, $760 \mathrm{~m}$, 29-06-2007, Fuertes \& Prada, MACB 105491. Salta: Orán, Reserva de San Andrés, selva pedemontana con helechos arborescentes, paraje de Las Maromas, $23^{\circ} 12^{\prime} \mathrm{S} 64^{0^{\prime}} 43^{\prime} \mathrm{W}, 650 \mathrm{~m}, 17-06-2008$, Fuertes, MACB 105492

Citas previas.-En el NE y área rioplatense, Kühnemann (1944) para la provincia de Buenos Aires; Stark (1987), Hübschmann (1986) y Drehwald (1995) la citan para Chaco y Corrientes; Stark (1987) para Formosa y Entre Ríos y Herzog (1952), Stark (1987) y Drehwald (1995) para Misiones. En el NO, Brotherus (1917) y Stark (1987) lo mencionan para Salta; Herzog (1952) y Stark (1987) para Tucumán.

Ecología y distribución.-Crece epífita, en las selvas prístinas del Dominio Amazónico. Neotropical: Felippone (1917) la citó para Uruguay y Stark (1987), para Bolivia, Brasil, Paraguay, Uruguay y Argentina. En la Argentina se comporta como especie mesófila, higrófila y esciófila, crece en los bosques cálidos y húmedos del NO (Jujuy, Salta, Catamarca, Tucumán) y del NE (Formosa, Misiones, Corrientes, Entre Ríos y Buenos Aires). Esta aportación es cita nueva para las provincias de Catamarca y Jujuy.

\section{Meteoriaceae}

Meteorium deppei (Hornsch. ex Müll. Hal.) Mitt.

Neckera deppei Hornsch. ex Müll. Hal. 1850.-Meteorium illecebrum Sull. 1861.-_ Pilotrichella deppei (Hornsch. ex Müll. Hal.) Besch. 1872._Papillaria deppei (Hornsch. ex Müll. Hal.) A. Jaeger 1875-76.

*Jujuy: Ledesma, P. Nac. de Calilegua, Paraje El Tigre, bosque montano de alisos, $23^{\circ} 44^{\prime} \mathrm{S} 64^{\circ} 55^{\prime} \mathrm{W}$, entre 1550-1700 m, 16-06-2008, Fuertes \& Prada, BA, MACB 104462. Misiones: Iguazú, P. Nac. Iguazú, entre la estación Boca del Diablo al Pozo, selva montana en la ribera del río Iguazú, 2536'S 54³4’ W, 100 m, 24-06-2008, Fuertes \& Prada, MA, MACB104459; ibidem, entre la estación Boca del Diablo y

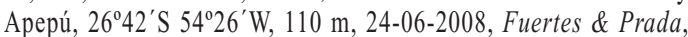
MACB104458; Manuel Belgrano, P. Nac. de Urugua-í, ruta 12, hacia Comandante Andresito, selva tropical en la ribera del arroyo Uruguayi, 25⒊ $36^{\prime} \mathrm{S} 54^{\circ} 34^{\prime} \mathrm{W}, 150-300 \mathrm{~m}, 10-07-2007$, Fuertes, BA, MACB1067259; San Pedro, Parque Regional Cruce Caballero, selva mixta de laurel, guatambú, pino paraná (Araucaria angustifolia) y helechos arbóreos de los géneros Dicksonia y Cyathea, $26^{\circ} 42^{\prime} \mathrm{S} 53^{\circ} 57^{\prime} \mathrm{W}$, entre 500-590 m, 14-09-2008, Fuertes, Oliván \& Prada, MACB104460, 105496. Salta: Orán, Reserva de San Andrés, selva pedemontana; paraje de Las Maromas $23^{\circ} 12^{\prime} \mathrm{S} 64^{0^{\prime}} 43^{\prime} \mathrm{W}$, entre 590-950 m, 17-06-2008, Fuertes, MACB 104457; Santa Victoria, P. Nac. de Baritú, pr. Los Toldos, bosque montano de Myrtaceae, $22^{\circ} 18^{\prime}$ S 64 $69^{\circ} 9^{\prime} \mathrm{W}$, entre 1590-1640 m, 28-06-2007, Fuertes \& Prada, BA, MACB105495; Reserva Nacio-

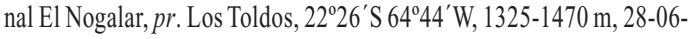
2007, Fuertes \& Prada, MA, MACB 105456, NY.

Citas previas.-En el NE, Hübschmann (1986) lo menciona para Corrientes y Herzog (1952) para Misiones. En el NO se ha recolectado en Salta y Tucumán (Tropicos.org 2014).

Ecología y distribución.-Epífita, común en la selva montana, también orófila (Andes tropicales). Neotropical: América del Norte (México), América Central, Sudamérica (Bolivia, Brasil, Argentina) (Tropicos.org 2014). Aquí se cita por primera vez para Jujuy, en Salta para el Departamento de Santa Victoria y en Misiones para los Departamentos General Manuel Belgrano y San Pedro.

Meteorium nigrescens (Hedw.) Dozy \& Molk.

Hypnum nigrescens Hedw. 1801.- Neckera nigrescens (Hedw.) Sm. ex Schwägr. 1828.- Cryphaea nigrescens (Hedw.) Mont. 1845.- 
Trachypus nigrescens (Hedw.) Mitt. 1864.—Papillaria nigrescens (Sw.) Jäger 1875-1876.

Misiones: San Pedro, Parque Regional Cruce Caballero, selva mixta de laurel, guatambú, pino paraná (Araucaria angustifolia) y helechos arbóreos (Cyathea), 26을 ${ }^{\prime} \mathrm{S} 53^{\circ} 57^{\prime} \mathrm{W}, 500-590 \mathrm{~m}, 14-09-2008$, Fuertes, Oliván \& Prada, MACB 105497; Guaraní, P. Nac. de Mocconá, selva tropical en la ribera del río Uruguay, $27^{\circ} 15^{\prime} \mathrm{S} 54^{\circ} 02^{\prime} \mathrm{W}, 450 \mathrm{~m}$, 27-09-2007, Fuertes, MACB 104455.

Citas previas.-Corrientes y Misiones (Hübschmann 1986); Salta (Hosseus 1938).

Ecología y distribución.-Epífita en troncos y ramas de bosques subtropical-tropicales. Neotropical: América del Norte (Estados Unidos y México), Centroamérica (Belice) y Sudamérica: Venezuela, Colombia, Ecuador, Perú, Bolivia, Brasil y Argentina (Tropicos. org 2014). Esta aportación es nueva para la brioflora de los departamentos de San Pedro y Guaraní (Misiones).

\section{Meteoridium remotifolium (Müll. Hal.) Manuel}

Leskea remotifolia Müll. Hal. 1846._- Neckera remotifolia (Müll. Hal.) Hornsch. ex Müll. Hal. 1851._-Meteoriopsis remotifolia (Müll. Hal.) Broth. 1906.

\begin{abstract}
*Jujuy: Ledesma, P. Nac. de Calilegua, paraje El Tigre, bosque montano con alisos, $23^{\circ} 44^{\prime} \mathrm{S} 64^{\circ} 55 \mathrm{~W}$, entre $1550-1700 \mathrm{~m}, 16-06-2008$, Fuertes \& Prada, BA, MACB104461, MA. *Misiones: Iguazú, P. Nac. de Iguazú, bañado, en la antigua mina Iguazú, $25^{\circ} 36^{\prime} \mathrm{S} 54^{\circ} 34^{\prime} \mathrm{W}, 170-220$ m, 11-07-2007, Fuertes \& Prada, MACB104464, M0; ibidem, selva montana, entre la estación Boca del Diablo al paraje El Pozo, $100 \mathrm{~m}$, 24-06-2008, Fuertes \& Prada, MACB 105717. Tucumán: Yerba Buena, selva montana de Myrtaceae, $p r$. Yerba Buena, 26 $49^{\prime} \mathrm{S} 65^{\circ} 19^{\prime} \mathrm{W}$, 950 m, 20-06-2007, Fuertes \& Prada, MA.
\end{abstract}

Citas previas.-Salta (Manuel 1977, Fuertes et al. 2011), Tucumán (Hosseus 1940, Hübschmann 1986, Fuertes et al. 2011) y Corrientes (Hübschmann 1986).

Ecología y distribución.-Epífita, en troncos y ramas de bosques húmedos. Neotropical, América del Norte, Sudamérica: Colombia, Venezuela, Guyana, Surinam, Ecuador, Perú, Bolivia, Brasil, Chile, Argentina y Uruguay (Manuel 1977). Se cita aquí como novedad para las provincias de Jujuy y Misiones, y para el Departamento Yerba Buena (Tucumán).

\section{Neckeraceae}

\section{- Pilotrichella flexilis (Hedw.) Ångström}

Leskea flexilis Hedw. 1801.- Hypnum flexile (Hedw.) Sw. ex Brid. 1801.- Isothecium flexile (Hedw.) Brid. 1827.-Neckera flexilis (Hedw.) Müll. Hal. 1850._- Meteorium flexile (Hedw.) Mitt. 1869.
Misiones: Iguazú, P. Nac. de Iguazú, sobre troncos y ramas de diferentes forófitos de la selva Paranaense, Estación del Palmito y Palo Rosa, $25^{\circ} 44^{\prime} \mathrm{S} 54^{\circ} 28^{\prime} \mathrm{W}, 160$ m, 13-09-2008, Fuertes, MACB 106718.

Ecología y distribución.- Epífita, en troncos y ramas, péndula. Pantropical. Se desarrolla en dos áreas disyuntas, África: Tanzania, Madagascar, Islas Mascareñas, y América: México, Belice, Venezuela, Colombia, Ecuador, Perú, Bolivia, Brasil (Tropicos.org 2014) y Argentina (esta aportación).

\section{- Porotrichodendron lindigii (Hampe) W. R. Buck}

Pilotrichum lindigii Hampe 1866.- Porotrichum lindigii (Hampe) Mitt. 1869._- Pterobryum lindigii (Hampe) Besch. 1894._- Pirea cavifolia Cardot \& Herzog 1911.- Porotrichum cavifolium (Cardot \& Herzog) Magill \& B. H. Allen 1992.

Salta: Los Monteros, entre la Quebrada de Scoipe y Los Laureles, epífita en troncos, en el bosque neotropical de las Yungas, $25^{\circ} 09^{\prime} \mathrm{S}$ $65^{\circ} 45^{\prime} \mathrm{W}, 1370 \mathrm{~m}, 15-06-2008$, Fuertes, Gallardo \& Prada, MACB 106714.

Ecología y distribución.-Epífita en diferentes forófitos de las yungas; se comporta como meso-higrófila. Neotropical, Centroamérica y Sudamérica: Venezuela, Colombia, Ecuador, Bolivia (Ireland \& Allen 2010) y Argentina (esta aportación).

\section{Porotrichodendron superbum (Taylor) Broth.}

Leskea superba Taylor 1846.- Hypnum superbum (Taylor) Müll. Hal. 1851.-Porotrichum superbum (Taylor) Hampe 1862.- Thamnium superbum (Taylor) Kindb. 1902.-Porotrichodendron robustum Broth. 1916.

*Salta: Santa Victoria, P. Nac. de Baritú, bosque montano de Myrtaceae, con Alnus acuminata, 22031'S 64²41'W, 1520-1720 m, 27-062007, Fuertes \& Prada, MACB 106368; ibidem, Reserva Nacional El Nogalar, $p r$ Los Toldos, bosque montano -en las Yungas- $22^{\circ} 18^{\prime} \mathrm{S}$

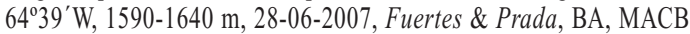
106367, MO; Orán, Reserva de San Andrés, selva pedemontana con helechos arborescentes, $23^{\circ} 12^{\prime} \mathrm{S} 64^{\circ} 43^{\prime} \mathrm{W}$, pr. Río Blanquito, 590-950 m, 17-06- 2008, Fuertes \& Prada, MACB 106716. Tucumán: Chicligasta, Sierra de Aconquija, selva montana de Myrtaceae con alisos, $27^{\circ} 20^{\prime} \mathrm{S} 65^{\circ} 48^{\prime} \mathrm{W}, 885 \mathrm{~m}, 22-06-2007$, Fuertes \& Prada, BA, LIL, MA, MACB 106364; ibidem, subiendo a La Banderita, 1450 m, 22-06-2007, Fuertes \& Prada, MACB 106365.

Citas previas.-Tucumán (Bartram 1965).

Ecología y distribución.-Epífita en troncos y ramas de selvas y bosques montanos, cálidos y húmedos. Neotropical. Norteamérica: México, Centroamérica, Sudamé- 
rica: Colombia, Ecuador, Perú, Bolivia y Argentina (Ireland \& Allen 2010). Es primera cita para la provincia de Salta y para los departamentos tucumanos de Chicligasta y Tafí del Valle.

\section{Porotrichum thieleanum (Müll. Hal.) Mitt.}

Hypnum thieleanum Müll.Hal. 1851.-Porotrichum thieleanum Müll. Hal. 1879.- Pilotrichum thieleanum (Müll. Hal.) Hampe 1879.Thamnium thieleanum (Müll. Hal.) Kindb. 1902._Pinnatella pinnatelloides (Müll. Hal.) Broth. ex Paris 1909.

Salta: Orán, Reserva de San Andrés, paraje Los Naranjos, selva pedemontana con maromas, $23^{\circ} 10^{\prime} \mathrm{S} 63^{\circ} 29^{\prime} \mathrm{W}, 400-650 \mathrm{~m}, 17-06-2008$, Fuertes \& Prada (MACB 106894); Santa Victoria, Reserva Nacional El Nogalar, $p r$. Los Toldos, bosque montano con alisos, entre 1590-

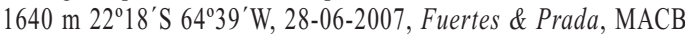
106368. *Tucumán: Chicligasta, Sierra de Aconquija, subiendo al paraje La Banderita, selva montana de Myrtaceae, con alisos -las Yungas- $27^{\circ} 18^{\prime} \mathrm{S} 65^{\circ} 54^{\prime} \mathrm{W}, 1450 \mathrm{~m}, 22-06-2007$, Fuertes \& Prada, MACB 1063659 .

Citas previas.-Salta, Orán, Río Seco, recolectada en 1873 por Lorentz, ejemplar designado como tipo, por Kindberg (1902) y nominado como Thamnium thieleanum. Churchill \& Schiavone la recolectan en San Andrés, Orán, en 1999 (Tropicos.org 2014).

Ecología y distribución.-Epífita, se comporta como especie mesófila, higrófila e indiferente edáfica. Neotropical. En Sudamérica se encuentra en Perú, Bolivia, Brasil y Argentina, donde es un taxón poco común, que se ha localizado en los bosques montanos más prístinos de Tucumán y Salta. Es novedad para la brioflora de Tucumán y para el Departamento de Santa Victoria (Salta).

\section{- Thamnobryum fasciculatum (Sw. ex Hedw.) I. Sastre}

Hypnum fasciculatum Sw. ex Hedw. 1801.- Porothamnium fasciculatum (Sw. ex Hedw.) M. Fleisch. 1808. - Neckera fasciculata (Sw. ex Hedw.) Arn. 1827.- Thamnium fasciculatum (Sw. ex Hedw.) Müll. Hal. 1898.

Jujuy: Ledesma, P. Nac. Calilegua, arroyo Tres Cruces, $23^{\circ} 44^{\prime} \mathrm{S}$ $64^{\circ} 55^{\prime} \mathrm{W}, 1060 \mathrm{~m}, 29-06-2007$, Fuertes \& Prada, BA, MACB 1063702; ibidem, camino de la Junta a la lagunilla, $23^{\circ} 44^{\prime} \mathrm{S} 64^{\circ} 55^{\prime} \mathrm{W}, 760 \mathrm{~m}, 29-$ 06-2007, Fuertes \& Prada, MA, MACB 106369; ibidem, paraje El Tigre, selva montana de Myrtaceae, 1550-1700 m, 16-06-2008, Fuertes \& Prada, MACB 106726; Manuel Belgrano, subida a las lagunas de Yala, bosque montano de Juglans australis y Alnus acuminata, $24^{\circ} 07^{\prime} \mathrm{S}$ $65^{\circ} 22^{\prime} \mathrm{W}, 1250-2000 \mathrm{~m}, 14-06-2008$, Fuertes \& Prada, BA, MACB 105507. Misiones: Iguazú, P. Nac. de Iguazú, cuenca alta del río Paraná, entre la Estación de Palmito y Palo Rosa, $25^{\circ} 42^{\prime} \mathrm{S} 54^{\circ} 26^{\prime} \mathrm{W}, 160 \mathrm{~m}$, 13-09-2008, Fuertes, Oliván, Herrero \& Prada, BA, MACB 106896;
Guaraní, Reserva de la Biosfera Yaboti, selva tropical a lo largo del arroyo Yabotí Guazú, $27^{\circ} 10^{\prime}$ S5 54 $04^{\prime} \mathrm{W}, 410 \mathrm{~m}, 27-09-2007$, Fuertes \& Prada, MACB 106695. Salta: Santa Victoria, Reserva Nacional El Nogalar, pr. Los Toldos, 22 ${ }^{\circ} 18^{\prime} \mathrm{S}$ 64³9'W, 1590-1640 m, 28-06-2007, Fuertes \& Prada, MACB 106371; ibidem, P. Nac. de Baritú, pr. Los Toldos, 1320-1470 m, 27-06-2007, Fuertes \& Prada, MACB 106373; Orán, Reserva de San Andrés, paraje Los Naranjos, selva pedemontana con abundancia de Ficus maroma, pr. Río Blanquito de Santa Cruz, $23^{\circ} 10^{\prime} \mathrm{S} 63^{\circ} 29^{\prime} \mathrm{W}, 400-650 \mathrm{~m}, 17-06-2008$, Fuertes, BA, MA, MACB 105340. Tucumán: Tafí del Valle, las yungas, en los alrededores de Vi-

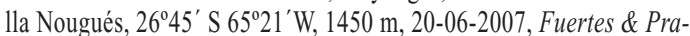
da, MACB106715; Chicligasta, Sierra de Aconquija, en troncos de diferentes forófitos, selva montana de Myrtaceae con Alnus acuminata, subiendo a La Banderita, 2720'S 654' W, 1450 m, 22-06-2007, Fuertes \& Prada, MACB 106372.

Ecología y distribución.- Epífita en troncos, base de troncos, tocones; se comporta como especie mesófila, higrófila, esciófila. Neotropical: Centroamérica y Sudamérica: Venezuela Colombia, Perú, Bolivia, Brasil (Allen \& Churchill 2002) y Argentina (esta aportación).

\section{Pilotrichaceae}

\section{Thamniopsis incurva (Hornsch.) W. R. Buck}

Chaetophora incurva Hornsch. 1820.- Lepidopilum incurvum (Hornsch.) Müll. Hal. 1848.-Callicostella incurva (Hornsch.) Ångström 1876.- Hookeria incurva (Hornsch.) Hook. \& Grev. 1895.-Hookeriopsis incurva (Hornsch.) Broth. 1907.

Misiones: Guaraní, Reserva de la Biosfera Yabotí, selva tropical con helechos arborescentes, a lo largo del arroyo Yabotí Guazú, pr. El Soberbio, $27^{\circ} 10^{\prime} \mathrm{S} 54^{\circ} 04^{\prime} \mathrm{W}, 410 \mathrm{~m}, 27-09-2007$, Fuertes \& Prada, MACB 105506; ibidem, selva tropical cerca del río Uruguay, $p r$. El Soberbio, $27^{\circ} 15^{\prime} \mathrm{S} 54^{\circ} 02^{\prime} \mathrm{W}, 450 \mathrm{~m}, 27-09-2007$, Fuertes \& Prada, BA, MACB 106724, NY.

Citas previas.- Hübschmann (1986) y Drehwald (1995) la citan para Misiones como Hoockeriopsis incurva. Matteri lo recolectó en 1969, en el Parque Nacional Iguazú (BA 18002).

Ecología y distribución.-Epífita en troncos y ramas de diferentes forófitos, mesófila y esciófila; forma extensas alfombras hacia la base de los troncos de árboles Neotropical. Buck (1987) lo menciona para México, Guatemala, Panamá, Trinidad, Cuba, Puerto Rico, República Dominicana, Guyana, Brasil y Argentina; además, se ha citado de Venezuela, Colombia y Paraguay (Tropicos. org 2014). En Argentina su área está restringida a Misiones; esta aportación es novedad para la brioflora del departamento de Guaraní. 


\section{Polytrichaceae}

- Atrichum polycarpum (Müll. Hal.) Mitt.

Catharinea polycarpa Müll. Hal. 1851.

Jujuy: Ledesma, P. Nac. Calilegua, selva montana de Myrtaceae - yungas, suelos ácidos, húmedos y sombríos, camino de la Junta a la lagunilla, 234' S 64'55'W, 760 m, 29-06-2007, Fuertes \& Prada, BA, MACB 106259. Salta: Capital, Barranca de San Lorenzo, en suelos húmedos del bosque montano de alisos, $24^{\circ} 44^{\prime} \mathrm{S}, 65^{\circ} 29^{\prime} \mathrm{W}, 1500 \mathrm{~m}, 30$ 07-2007, Fuertes \& Prada, BA, MACB 105485. Tucumán: Tafí del Valle, selva montana de Myrtaceae - yungas, suelos húmedos de la la-

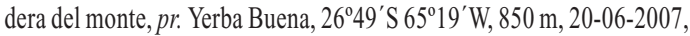
Fuertes \& Prada, MACB 105486; ibidem, en suelos húmicos, húmedos, alrededores de Villa Nougués, $26^{\circ} 45^{\prime} \mathrm{S} 65^{\circ} 21^{\prime} \mathrm{W}, 1450 \mathrm{~m}, 20-06$ 2007, Fuertes \& Prada, BA, MA, MACB 105484, MO, NY; ibidem, en rocas húmedas y cascadas al lado del puente, subiendo a San Javier, 1150 m, 20-06-2007, Fuertes \& Prada, MACB 106257; Chicligasta, Sierra de Aconquija, suelos húmedos cerca de arroyos, bosque montano con alisos- las Yungas-, subiendo a La Banderita, $27^{\circ} 42^{\prime} \mathrm{S} 65^{\circ} 54^{\prime} \mathrm{W}$, 1450 m, 22-06-2007, Fuertes \& Prada, MACB 106258.

Ecología y distribución.-Terrícola, suelos húmedos cerca de arroyos y veneros, cascadas, a veces sobre raíces; se comporta como mesófila y esciófila. Neotropical, amplia distribución en América: Norteamérica (México), Centroamérica y Caribe (Jamaica), Sudamérica (Colombia, Ecuador, Bolivia, Brasil (Nyholm 1971, Tropicos.org 2014) y Argentina (esta aportación).

\section{Pterobryaceae}

\section{Pterobryon densum Hornsch.}

Hypnum densum Sw. ex Hedw. 1801.-Pterogonium densum (Sw. ex Hedw.) Schwägr. 1828. - Pterobryum lorentzii Mül. Hal. 1879.- P. subangustifolium Müll. Hal. 1898._- Prionodon splendens Herzog 1909.

Salta: Santa Victoria, Reserva Nacional El Nogalar, pr. Los Toldos, en troncos, bosque montano, 22 $21^{\circ} \mathrm{S} 64^{\circ} 41^{\prime} \mathrm{W}, 1520-1720 \mathrm{~m}, 28-06-2007$, Fuertes \& Prada, MACB 106366.

Citas previas.- Müller (1879) describe los especímenes recolectados por Lorentz en 1873 (Salta, San Andrés) como Pterobryum lorentzii; en la misma localidad la recolectaron Churchill \& Schiavone en 1999 (Tropicos.org. 2014).

Ecología y distribución.-Epífita en troncos de diferentes forófitos; mesófila e higrófila. Neotropical, Norteamérica (México), Centroamérica y Caribe (Haití) y Sudamérica (Colombia, Bolivia, Brasil y Argentina)
(Tropicos.org 2014). Poco común en Argentina, es novedad para el Departamento de Santa Victoria (Salta) y tercera cita para Argentina.

\section{Pterobryopsis stolonacea (Müll. Hal.) Broth.}

\section{Pterobryum stolonaceum Müll. Hal. 1879)}

Salta: Orán, Reserva de San Andrés, selva pedemontana, paraje de Las Maromas, $23^{\circ} 07^{\prime} \mathrm{S} 64^{\circ} 37^{\prime} \mathrm{W}, 650 \mathrm{~m}$, pr. Río Blanquito de Santa Cruz, 890-950 m, 17-06-2008, Fuertes, Martínez \& Prada, BA, MACB 105342.

Citas previas.- En el NO, Müller (1878-1879) lo menciona para Jujuy y Herzog (1952) para Tucumán. Ha sido recolectada en Salta (Tropicos. org, 2014). En el NE, Hübschmann (1986) lo menciona para Corrientes.

Ecología y distribución. - Usualmente epífita en troncos y base de troncos, tocones, a veces saxícola. Se comporta como especie mesófila, higrófila y esciófila. Neotropical, hasta ahora restringida a los bosques húmedos de climas cálidos del NO de Sudamérica, en áreas montanas de Perú, Bolivia y Argentina (Tropicos.org. 2014).

\section{Racopilaceae}

Racopilum tomentosum (Hedw.) Brid.

Hypnum tomentosum Hedw. 1801.- Racopilum mnioides P. Beauv. 1805.-Hookeria tomentosa (Hedw.) Arn. 1823.-Hypopterygium tomentosum (Hedw.) Müll. Hal. 1850.

Jujuy: Ledesma, P. Nac. de Calilegua, selva montana de Myrtaceae yungas-, sobre troncos de árboles, con Pilosium sp, y otros musgos pleurocárpicos, $23^{\circ} 44^{\prime} \mathrm{S} 64^{\circ} 55^{\prime} \mathrm{W}, 760 \mathrm{~m}, 29-06-2007$, Fuertes \& Prada, MACB 106320, 106322. Misiones: Guaraní, Reserva de la Biosfera Yabotí, selva tropical con helechos arborescentes, arroyo Yabotí Guazú, pr. El Soberbio, 27ำ10'S, 540.' W, 410 m, 27-09-2007, Fuertes \& Prada, BA, MA, MACB 105500. Salta: Orán, Reserva de San Andrés, selva pedemontana con helechos arborescentes, paraje Las Maromas, $23^{\circ} 07^{\prime} \mathrm{S} 64^{\circ} 37^{\prime} \mathrm{W}, 650-950 \mathrm{~m}, 17-06-2008$, Fuertes, Martinez \& Prada, MACB 105499. Tucumán: Yerba Buena, selva montana de Myrtaceae -Yungas- en suelos húmedos del bosques de Alnus acumi-

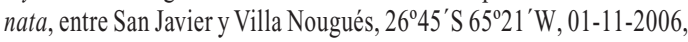
Fuertes, BA, MACB 106325.

Citas previas.- Hosseus (1940) y Hübschmann (1986) lo citan en el NE de la Argentina, (Misiones y Corrientes). Al NO de la Argentina, Brotherus (1917) la recolecta en Jujuy; Herzog (1952) en Salta y en Tucumán Herzog (1952). 
Ecología y distribución.-Epífita, a veces saxícola, meso-esciófila e higrófila. Neotropical, crece en zonas sombrías de bosques húmedos de América, desde México hasta Bolivia, Brasil, Chile y Argentina. Estas aportaciones son primeras citas para los Departamentos de Ledesma (Jujuy), Yerba Buena (Tucumán) y Guaraní (Misiones).

\section{Rigodiaceae}

Rigodium toxarion (Schwägr.) A. Jäger

Hypnum toxarion Schwägr. 1816.-H. solutum Taylor 1846.- H. argentinicum Müll. Hal. 1872.- Rigodium argentinicum (Müll. Hal.) Kindb. 1891.

Tucumán: Chicligasta, Sierra de Aconquija, paraje La Banderita, bosque montano con alisos (Alnus acuminata), 27 $7^{\circ} 18^{\prime} \mathrm{S} 65^{\circ} 54^{\prime} \mathrm{W}, 1950 \mathrm{~m}$, 22-06-2007, Fuertes \& Prada, MACB 105501, S.

Citas previas.- Müller (1882) describe Hypnum argentinicum a partir del material recolectado por Lorentz en 1872, en Las Juntas (Tucumán), ejemplar designado como lectótipo por Zomlefer (1993).

Ecología y distribución.- Epífita, mesófila e higrófila, crece en forófitos perennifolios. Pantropical. En el Paleotrópico crece en África (Tanzania, Mozambique, Zimbabue, Sudáfrica y Madagascar). En el Neotrópico se menciona para México, Costa Rica, El Salvador, Guatemala, Panamá, Haití, República Dominicana, Venezuela, Colombia, Ecuador, Perú, Bolivia, Brasil, Chile, Argentina y Uruguay (Zomlefer 1993). En Argentina, crece en troncos y ramas de árboles de bosques cálidos y húmedos de las yungas, entremezclada con otras muscíneas. Esta aportación es segunda cita para Argentina y primera cita para Chicligasta (Tucumán).

\section{Stereophyllaceae}

\section{Juratzkaea argentinica (Thér.) W. R. Buck}

Juratzkaea seminervis var. argentinica Thér. 1936.-Juratzkaea seminervis var. argentinica fo. robustior Thér. ex Hosseus 1937.- Juratzkaea seminervis subsp. argentinica (Thér.) W. R. Buck 1977.

**Salta: Los Monteros, base de troncos, entre la Quebrada de Scoipe y Los Laureles, $25^{\circ} 09^{\prime} \mathrm{S} 65^{\circ} 45^{\prime} \mathrm{W}, 1370 \mathrm{~m}, 15-06-2008$, Fuertes, Gallardo \& Prada, MACB 105343.

Citas previas.- Hosseus (1938) lo recolectó en Córdoba (San Agustín) y lo describió como fo. robustior. Herzog (1952) lo citó para Córdoba y Salta.
Ecología y distribución.-Forma pequeñas alfombrillas laxas, sobre piedras y base de troncos; se comporta como especie mesófila, higrófila, con tendencia nitrófila. Neotropical, endémica de Argentina (Ireland \& Buck 1994). Esta aportación es nueva para el departamento Los Monteros, segunda cita provincial (Salta) y tercera cita para la Argentina.

- Pilosium chlorophyllum (Hornsch.) Müll. Hal. ex Broth

Hypnum chlorophyllum Hornsch 1840.- Hypnum cruegerianum Müll. Hal. 1875.- Stereophyllum cruegerianum Paris 1897.— S. subchlorophyllum Paris 1898.

Jujuy: Ledesma, P. Nac. de Calilegua, selva montana de Myrtaceae, camino de la Junta a la lagunilla, $23^{\circ} 44^{\prime} \mathrm{S} 64^{\circ} 55^{\prime} \mathrm{W}, 800 \mathrm{~m}, 29-06-2007$, Fuertes \& Prada, BA, MACB 106722, 104740, MA. Salta: Orán, San Andrés, selva pedemontana con helechos arborescentes, Las Maromas pr. Río Blanquito de Santa Cruz, 230 $07^{\prime} \mathrm{S} 64^{\circ} 37^{\prime} \mathrm{W}, 890-950$ m, 17-062008, Fuertes, Martínez \& Prada, MACB105339, MO, NY. Tucumán: Tafí del Valle, bosque montano, alrededores de Villa Nougués, $26^{\circ} 45^{\prime} \mathrm{S}$ $65^{\circ} 21^{\prime} \mathrm{W}, 1450 \mathrm{~m}, 20-06-2007$, Fuertes \& Prada, MACB 106723.

Ecología y distribución.-Epífita, principalmente hacia la base de troncos de árboles perennifolios; se comporta como especie mesófila e higrófila. Neotropical. México, Nicaragua, Costa Rica, Panamá, República Dominicana, Venezuela, Colombia, Ecuador, Perú Bolivia, Guyana, Surinam, Brasil, (Ireland \& Buck 1994), y Argentina (esta aportación).

\section{Stereophyllum radiculosum (Hook.) Mitt.}

Hookeria radiculosa Hook. 1818.- Pterygophyllum radiculosum (Hook.) Brid. 1827.-Stereophyllum rivulare Mitten. 1860.-Hypnum radiculosum Müll. Ha1. 1874.- Stereophyllum argentinicum Broth. 1917.

**Jujuy: Ledesma, P. Nac. Calilegua, ruta de la Junta a la lagunilla. $23^{\circ} 46^{\prime} \mathrm{S} 64^{\circ} 45^{\prime} \mathrm{W}, 800 \mathrm{~m}, 06-05-2007$, Prada \& Gallardo (MACB 105339, MO, NY).

Citas previas.-Ireland \& Buck (1994) lo mencionan para Jujuy (NO de la Argentina) y Formosa (NE de la Argentina).

Ecología y distribución.-Epífita, sobre troncos y ramas, se comporta como especie mesófila e higrófila, en bosques cálidos y húmedos. Pantropical, en áreas disyuntas de América, África, Asia (India) y Australia (Ireland $\&$ Buck 1994). En Argentina crece epífita en los bosques 
montanos de las Yungas. Aquí se cita por primera vez para Ledesma (Jujuy), es la segunda cita para la provincia de Jujuy y tercera referencia para la Argentina.

\section{Trachypodaceae}

\section{- Pseudotrachypus martinicensis (Broth.) Buck}

Papillaria martinicensis Broth. 1903._-Aerobryopsis martinicensis (Broth.) Spessard-Schueth 1994.

Misiones: Iguazú, P. Nac. de Iguazú, ribera del Arroyo de Santo Domingo, junto a la ruta $101,25^{\circ} 42^{\prime} \mathrm{S} 54^{\circ} 26^{\prime} \mathrm{W}, 90 \mathrm{~m}, 12-09-2008$, Fuertes, Oliván, Herrero, \& Prada, BA, MACB 114452, NY; entre Boca del Diablo y Apepú, selva montana de las riberas del Iguazú, 110 m, 2406-2008, Fuertes, MACB 104453.

Ecología y distribución.-Epífita en troncos y ramas, en bosques húmedos. Neotropical. Ireland \& Buck (1994) lo citan y recolectan para la República Dominicana. En Argentina, crece epífita en forófitos de bosques ribereños; su área de distribución está restringida a la provincia biogeográfica Paranaense (NE).

\section{CONCLUSIONES}

El catálogo presentado comprende 32 briófitos, pertenecientes a 15 familias, procedentes de los bosques montanos de las Yungas del noroeste de la Argentina y de la selva Misionera, provincia de Misiones, en el nordeste del país. Con esta aportación se ha ampliado el área de distribución de los taxones, tanto a nivel nacional como provincial 0 departamental.

Excepto Atrichum polycarpum, que presenta crecimiento acrocápico, forma céspedes cortos, más o menos densos y crece en suelos pedregosos, taludes y laderas del bosque, el resto de los taxones está representado por musgos pleurocárpicos, la mayoría epífitos, que forman extensas alfombrillas o tramas sobre troncos y ramas; frecuentemente tienen gametófitos largos y péndulos y son característicos de los hábitats más prístinos de selvas y bosques húmedos de las Yungas, en las provincias de Jujuy, Salta, Catamarca y Tucumán al NO y en la selva Misionera de la provincia de Misiones al NE de la Argentina.

Ocho especies: Atrichum polycarpum, Chryso-hypnum elegantulum, Pilosium chlorophyllum, Pilotrichella flexilis, Porotrichodendron lindigii, Pseudotrachypus martinicensis, Steerecleus scariosus y Thamnobryum fascicula- tum son nuevas aportaciones para la flora briológica argentina (Tabla 1).

Forsstroemia coronata es primera cita para las provincias de Catamarca y Jujuy. Braunia imberbis y Squamidium brasiliense son novedad para la brioflora de la provincia de Catamarca; Porotrichodendron superbum es primera cita para Salta y Schoenobryum concavifolium es nuevo taxón para la flora briofítica de Misiones. Cinco taxones: Aerolindigia capillacea, Braunia reflexifolia, Chryso-hypnum diminutivum, Meteorium deppei y Meteoridium remotifolium, son nuevas citas a la brioflora de la provincia de Jujuy (Tabla 1).

Se destaca, por su interés corológico, la presencia de taxones poco frecuentes en la brioflora de algunas provincias argentinas: en Jujuy, Braunia exserta, Cryphaea racomitrioides, Racopilum tomentosum y Stereophyllum radiculosum son segunda cita para la provincia y el último es, además, tercera cita para Argentina.

Juratzkaea argentinica, Meteorium deppei y Pterobryopsis stolonacea, son segunda cita para la provincia de Salta; Porotrichodendron superbum y Rigodium toxarion, son segundas citas para la flora de Tucumán. Thamniopsis incurva, citada únicamente en el Parque Nacional de Iguazú (Misiones), es la segunda cita para la provincia y para la Argentina.

Algunas especies del catálogo se citan por primera vez para algunos departamentos de ambas áreas, NO y NE, por lo que amplían notablemente, en ciertos casos, su área de distribución provincial.

La mayoría de las especies estudiadas son más frecuentes en las Yungas (NO argentino) como Aerolindigia capillacea, Atrichum polycarpum, Braunia exserta, B. imberbis, B. reflexifolia, Cryphaea racomitrioides, Chrysohypnum elegantulum, Pilosium chlorophyllum, Porotrichodendron superbum, Rigodium toxarion, Steerecleus scariosus y Stereophyllum radiculosum; otras presentan una disyunción neotropical, las Yungas-selva Paranaense, como Chryso-hypnum diminutivum, Erythrodontium squarrosum, Meteorium deppei, M. nigrescens, Meteoridium remotifolium, Mittenotamnium reptans, Pterobryopsis stolonacea, Racopilum tomentosum, Squamidium brasiliense y Thamnobryum fasciculatum y otras, como PilotricheIla flexilis, Pseudotrachypus martinicensis y Thamniopsis incurva, tienen su área de distribución restringida a la selva Paranaense argentina (ver Tabla 1).

\section{AGRADECIMIENTOS}

Las autoras expresan su agradecimiento a Olga Martínez y Lázaro Juan Novara, de la Universidad de Salta, a María Magdalena Schiavone, 
de la Universidad de Tucumán, a Luis Ariza de la Universidad Nacional de Córdoba y a Andrea Coradeghini, del Herbario del Museo de Ciencias Naturales Bernardino Rivadavia, de la Ciudad Autónoma de Buenos Aires, por las atenciones y facilidades recibidas para la consulta y estudio del material de los herbarios de esas Instituciones científicas. Nuestro reconocimiento también a los directores y responsables de los parques y reservas naturales que se citan en el texto por la información y los permisos preceptivos que nos dispensaron para estudiar la brioflora argentina. Esta investigación ha sido subvencionada por el Ministerio de Asuntos Exteriores del Reino de España, a través de los Proyectos AECIA-3818-2005, A-6307-2006, A- 8930-2007 y del Ministerio de Ciencia e Innovación del Reino de España, por el Proyecto CGL2009-13622.

\section{BIBLIOGRAFÍA}

Allen, B. H. \& Churchill, S. R. 2002. A new species of Thamnobryum (Musci, Neckeraceae). Novon 12: 155-158.

Allen, B. H. \& CrosbY, M. R. 1986. Revision of the genus Squamidium (Musci: Meteoriaceae). J. Hattori Bot. Lab. 61: 423476.

BARTRAM, E. B. 1965. New and noteworthy mosses from northern Argentina. Rev. Bryol. 33 (3-4): 323-327.

Biasuso, A. B. 1992. El género Braunia (Hedwigiaceae, Musci) en Tucumán. Lilloa 38: 5-21.

Brotherus, V. F. 1917. Contributions à la flore bryologique de l'Argentina. Arkiv für Bot. 15 (1-2):1-15.

Buck, W. R. 1987. Taxonomic and nomenclatural rearrangements in the Hookeriales with notes on West Indian taxa. Brittonia 39: 210-224.

CABRERA, A. L. 1976. Regiones fitogeográficas argentinas. En W. F. Kugler (Ed.), Enciclopedia argentina de agricultura y jardinería, 2 (2a ed.). ACME. Buenos Aires, Argentina.

Churchill, S. R. \& Linares, E. L. 1995. Prodomus Bryologie Novo-Granatensis. Edit. Guadalupe Ltda. Santa Fé de Bogotá, Colombia.

DrehwaLd, U. 1995. Epiphytische Pflanzengesellschaften in NO-Argentinien. Dissertattione Botanischen 250: 1-175.

Felippone, F. 1917. Contribution a la flore bryologique de $L^{\prime}$ Uruguay, $3^{\circ}$ fasc.: 40-45. Montevideo, Mosca Hnos.

Fuertes E. 2008. New national and regional records. 19. Orthostichopsis tetragona (Hedw.) Broth. in Argentina. J. Bryol. 30(3): 233.

Fuertes, E. \& HedENÄs, L. 2007. New national and regional records. 16. Warnstorfia hipichensis (R. S. Williams) Hedenäs for Argentine. J. Bryol. 29(3): 201.

Fuertes, E.; Jiménez, S. \& Oliván, G. 2012. Contribution to the bryological flora of Argentina. Sphagnum perichaetiale Hampe and S. recurvum P. Beauv. (Sphagnaceae). Bryologist 115(2):178-182.

FueRTES, E. \& MARCHESSI, J. 2013. Notula taxonómica, nomenclatural y corológica de Steerecleus (Bryophyta: Brachytheciaceae) en Uruguay. Bot. Complut. 37: 35-39.

Fuertes, E. \& Oliván, G. 2008. Revisión de Plagiomnium (Plagiomniaceae, Bryopsida) en Argentina. Tropical Bryol. 29: 60-70.

Fuertes, E. \& Rodriguez, M. 2008. Aportaciones a la flora briológica argentina. Notula I. Bot. Complut. 32: 75-83.

Fuertes, E.; San Martín, C. \& Escobar, L. 2011. Zelometeorium y Meteoridium (Bryophyta, Brachytheciaceae) en Argentina. Bot. Complut. 35: 27-37

Goffinet, B.; Buck, W. R. \& SHaw, A. 2008. Morphology, anatomy and classification of the Bryophyta. En: B. Goffinet
\& A. Shaw (Eds.), Bryophyte biology: 55-138. Cambridge University Press.

Gradstein, S. R.; Churchill, S. P. \& Salazar-Allen, N. 2001. Guide to the bryophytes of tropical America. Mem. New York Bot. Gard. 86: 1-577.

HerTER, W. G. 1943. Plantae uruguayenses novae vel criticae. Pars IV. Rev. Sudamericana Bot. 7(6-8): 171-260.

HerzoG, TH. 1952. Beiträge zur Kenntnis der argentinischen Bryophytenflora. Feddes Repert. 30, XII: 1-27.

Hosseus, C. C. 1938. Musgos de la república Argentina. Arch. Escuela de Farmacia, Fac. Cienc. Médic. Córdoba (R. A.) 8: 3-58.

Hosseus, C. C. 1940. Musgos del territorio de Misiones, nuevos para Argentina. Bol. Ingeniería 3: 1-29.

Ireland, R. R. \& Allen, B. H. 2010. Porotrichodendron. En: B. H. Allen (Ed.), Moss flora of Central America. Part. 3. Anomodontaceae-Symphodontaceae, Monogr. Syst. Bot. Missouri Bot. Gard. 117: 315-325.

Ireland, R. R. \& Buck, W. R. 1994. Stereophyllaceae. Flora Neotropica, Monograph 65: 1-50.

KINDBERG, N. C. 1902. Grundziger einer Monographie der Laubmoos-Gattung Thamnium. Hedwigia 41(5): 225-268.

Koponen, T. \& FuerTes E. 2010. Contribution to the bryological flora of Argentina. II. Rhodobryum (Bryaceae, Musci). Bryologist 113(1): 132-143.

KühnemanN, O. 1944. Géneros de briófitas de los alrededores de Buenos Aires. Lilloa 10: 1-232.

Manuel, M. G. 1977. The genus Meteoridium (C. Müll.) Manuel, stat. nov. (Bryopsida, Meteoriaceae). Lindbergia 4(12): $45-56$.

MatTeRI, C. M. 2003. Los musgos (Bryophyta) de Argentina. Tropical Bryol. 24: 33-100.

Menzel, M. 1991. A taxonomic review of the genera Lindigia Hampe (Meteoriaceae, Leucodontales) and Aerolindigia gen. nov. (Brachytheciaceae, Hypnales), Bryopsida. Nova Hedwigia 52 (3-4): 319-335.

MÜLLER, C. 1878-1879. Prodromus bryologiae Argentinicae. Linnaea 42: 217-460

MÜLLER, C. 1882. Prodromus bryologiae Argentinicae. Linnaea 43: 341-486.

MüBschmanN, A. 1986. Bryologische Studien in Nordargentinien. Veröf. Geobot. Inst. ETH. Stiftung Rübel, Zürich 91: 305-317.

Nishimura, N \& Hisatsugu, A. 1994. Chryso-hypnum Hampe. En: A. J. Sharp, H. Crum \& P. M. Eckel, (Eds.), The moss flora of Mexico. Mem. New York Bot. Gard. 2: 1053-1055. 
NyнoLm, E. 1971. Studies in the genus Atrichum P. Beauv. Lindbergia 1-2: 1-33.

PurselL, R. A. 1973. Un censo de los musgos de Venezuela. Bryologist 76(4): 473-500.

RA0, P. 2001. Monographic studies on Cryphaea. Bryobrothera 6: 65-42

RoBinson, H. 1987. Notes on generic concepts in the Brachytheciaceae and the new genus Steerecleus. Mem. New York Bot. Garden 45: 678-681.

Sharp, A. J.; CRum, H. \& Eckel, P. M. 1993. The moss flora of Mexico. Mem. New York Bot. Garden, 69, 2 vols.
StaRK, L. R. 1987. A taxonomic monograph of Forsstroemia Lindb. (Bryopsida, Leptodontaceae). J. Hattori Bot. Lab. 63: 133-218.

Suarez, G. M. \& Schiavone, M. M. 2010. La familia Cryphaeaceae (Briófita) en los bosques del noroeste de Argentina. Bol. Soc. Bot. Argentina 45(1-2): 29-45.

Tropicos. ORg. 2014. Missouri Botanical Garden. 15 marzo 2014. (http:www.tropicos.org).

Zomlefer, W. B. 1993. A revision of Rigodium (Musci, Rigodiaceae). Bryologist 96: 1-72. 